\title{
Probing the Dirac or Majorana nature of the heavy neutrinos in pure leptonic decays at the LHC
}

\author{
Carolina Arbelaéz, ${ }^{*}$ Claudio Dib, ${ }^{\dagger}$ Iván Schmidt, ${ }^{*}$ and Juan Carlos Vasquez ${ }^{\S}$ \\ Universidad Técnica Federico Santa María, \\ Centro-Científico-Tecnológico de Valparaíso, Valparaíso 2390123, Chile
}

(Received 2 January 2018; published 7 March 2018)

\begin{abstract}
We propose a strategy for distinguishing the Dirac/Majorana character of heavy neutrinos with masses below the $W$-boson mass, using purely leptonic decays at the LHC. The strategy makes use of a forwardbackward asymmetry of the opposite-charge lepton in the $W^{+} \rightarrow l^{+} l^{+} l^{\prime} \nu$ decay. In order to check the experimental feasibility of the model, we show-through a numerical analysis and for different ranges of the heavy neutrino masses- that in the decay $W^{+} \rightarrow e^{+} e^{+} \mu^{-} \nu$ one can distinguish the positron from the $W$ decay from the positron coming from the heavy neutrino. Finally, we estimate the number of events at the LHC Run II for a Dirac and Majorana $N$ neutrino with an integrated luminosity of $120 \mathrm{fb}^{-1}$. Signals can be found if heavy-to-light neutrino mixings are $\left|U_{N \mu}\right|^{2},\left|U_{N e}\right|^{2} \gtrsim 10^{-6}$.
\end{abstract}

DOI: 10.1103/PhysRevD.97.055011

\section{INTRODUCTION}

Experiments in the last decades have confirmed that at least two of the three known neutrinos must have nonzero masses, and that all three have nontrivial mixings with respect to electroweak flavors. Ultimate confirmation of neutrino masses came from the results of atmospheric, solar, and reactor neutrino experiments [1-4]. This evidence, compounded by the fact that the neutrino masses happened to be tiny with respect to the other Standard Model (SM) fermions, is currently an outstanding theoretical path for physics that goes beyond the SM. Most of the explanations of the neutrino masses' smallness are based on the existence of extra heavy neutral fermions, which could be Dirac or Majorana [5]. In fact, the most widely accepted mechanism to generate small neutrino masses is the seesaw mechanism [6-10], which involves extra heavy sterile neutrinos (henceforth denoted generically by $N$ ). Moreover, in most of these scenarios the neutrinos are Majorana instead of Dirac fermions, and this discrimination about their nature is a crucial and challenging piece of information that experiments must elucidate.

\footnotetext{
*carolina.arbelaez@usm.cl

†claudio.dib@usm.cl

*ivan.schmidt@usm.cl

§uan.vasquezcar@usm.cl
}

Published by the American Physical Society under the terms of the Creative Commons Attribution 4.0 International license. Further distribution of this work must maintain attribution to the author(s) and the published article's title, journal citation, and DOI. Funded by SCOAP ${ }^{3}$.
Direct collider searches for sterile heavy neutrinos may provide a simultaneous probe of both their Dirac or Majorana nature, as well as their mixing with the active neutrinos. At hadron colliders, a strong signal of heavy Majorana neutrinos has been shown to exist and it is the same-sign dilepton final states, with two jets and no missing transverse energy: $p p \rightarrow W \rightarrow N e^{ \pm} \rightarrow e^{ \pm} e^{ \pm} j j$. This was originally proposed in Ref. [11] and further studied in Refs. [12-21]. Using this channel, both the CMS and ATLAS experiments at the LHC have set direct limits on the light-heavy neutrino mixing [22-25] for masses of $m_{N} \sim 100-500 \mathrm{GeV}$.

For neutrino masses in the region $m_{N}<m_{W}$, the produced jets in the final state may not pass the cuts required to reduce the backgrounds, so the purely leptonic channels (such as $p p \rightarrow e^{ \pm} e^{ \pm} \mu^{\mp} \nu$ ) in which $\nu$ could be either a neutrino or an antineutrino may be more favorable [26]. Here we will focus on the signal $W^{ \pm} \rightarrow e^{ \pm} e^{ \pm} \mu^{\mp} \nu^{-}$, which would indicate the existence of a sterile neutrino through the subprocess $W^{ \pm} \rightarrow e^{ \pm} N$, followed by $N \rightarrow e^{ \pm} \mu^{\mp} \nu$. Notice that in the leptonic channel it is not clear a priori how to distinguish Dirac neutrinos from Majorana ones, since the final neutrino is undetected in both cases (i.e., the observed final states are $e^{ \pm} e^{ \pm} \mu^{\mp}$ or $\mu^{ \pm} \mu^{ \pm} e^{\mp}$ plus missing energy). Hence, an obvious question is whether a Dirac or Majorana $N$ can be distinguished at the LHC in these pure leptonic modes.

This has been answered in Refs. [27-30], where it was shown that the Dirac and Majorana cases could in fact be distinguished, depending on the relative size of the mixing between the $e$ or $\mu$ flavors and the heavy neutrinos. In particular, they have addressed the issue of Dirac/Majorana 
discrimination using the muon spectrum and a multivariable analysis.

Here we present a simpler approach that makes use of the forward-backward asymmetry proposed in Refs. [24,31]. Complementary to the strategy presented in Refs. [28-30], we find that a suitable quantity to discriminate between Dirac and Majorana in the purely leptonic channels is the forward-backward asymmetry of the unlike-charged lepton [14]. This strategy works even when the mixing of the heavy neutrinos with the charged leptons are equal or of the same order of magnitude.

The paper is organized as follows. In Sec. II we start by recalling the basic facts and some kinematical considerations involving the benchmark processes $W^{ \pm} \rightarrow e^{ \pm} e^{ \pm} \mu^{\mp} \nu$, followed by the subprocesses $W^{ \pm} \rightarrow e^{ \pm} N$ and $N \rightarrow e^{ \pm} \mu^{\mp} \nu$. We analyze a specific forward-backward asymmetry for the lepton-number-conserving (LNC) mode $W^{+} \rightarrow$ $e^{+} e^{+} \mu^{-} \nu_{e}$ and the lepton-number-violating (LNV) mode $W^{+} \rightarrow e^{+} e^{+} \mu^{-} \bar{\nu}_{\mu}$. This asymmetry allows to distinguish between Dirac and Majorana neutrinos in these purely leptonic decays at the LHC. In Sec. III we discuss the distinction of the two same-sign charged leptons in the final state at the LHC, which is crucial in order to extract the asymmetry from the experiment. In Sec. IV we conclude with a discussion of our results.

\section{THE DECAY RATES AND THE FORWARD-BACKWARD ASYMMETRY}

In this section we present the theoretical expressions for the purely leptonic processes $W^{ \pm} \rightarrow \ell^{ \pm} \ell^{ \pm} \ell^{\prime \mp} \nu$, the forward-backward asymmetry of the unlike-charge lepton $\ell^{\prime \mp}$, and the analysis that indicates that this asymmetry should be related to the Dirac/Majorana nature of the intermediate neutrino $N$.

The part of the Lagrangian relevant for this process is given by

$$
\mathcal{L}=\frac{g}{\sqrt{2}} \sum_{l=e, \mu \tau} W_{\mu} \bar{N}^{c} U_{N l}^{\dagger} \gamma^{\mu} P_{L} l+\text { H.c. }+\cdots,
$$

where $P_{L}=\left(1-\gamma_{5}\right) / 2, U_{l N}$ is the mixing matrix of the charged leptons, $l=e, \mu, \tau$, and $N$ denotes the heavy neutrino.

For simplicity, let us focus on the mode $W^{+} \rightarrow e^{+} e^{+} \mu^{-} \nu$; the modes with other flavors and charges are similar. Figure 1 shows the two possible processes for this mode, considering that the final neutrino $\left(\bar{\nu}_{\mu}\right.$ or $\left.\nu_{e}\right)$ is not observable. The upper and lower diagrams correspond to a LNC and a LNV process, respectively.

The rates of these two processes, in the notation of Fig. 1 and neglecting the masses of the final leptons, can be given in the narrow-width approximation for $N$ as
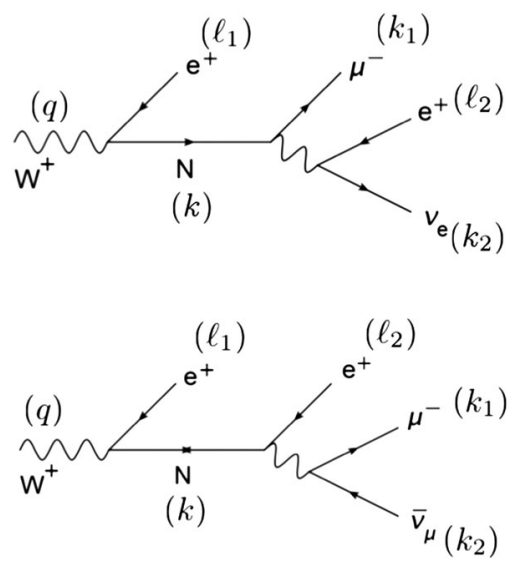

FIG. 1. Top: LNC decay of a $W^{+}$mediated by either a Dirac or Majorana neutrino $N$. Bottom: LNV decay of a $W^{+}$mediated by a Majorana neutrino only. The momenta are denoted by the symbols in parentheses.

$$
\begin{aligned}
\Gamma\left(W^{+}\right. & \left.\rightarrow e^{+} e^{+} \mu^{-} \nu_{e}\right)_{(L N C)} \\
= & \frac{64 \sqrt{2} G_{F}^{3}}{3} \frac{M_{W}^{5}}{m_{N} \Gamma_{N}} \\
& \times\left|U_{N e} U_{N \mu}\right|^{2} \int d \Phi_{2} \int d \Phi_{3} \frac{\left(k_{1} \cdot k_{2}\right)}{\left(M_{W}^{2}-m_{N}^{2}+2 k \cdot k_{1}\right)^{2}} \\
& \times\left\{2\left(k \cdot \ell_{2}\right)\left[\left(k \cdot \ell_{1}\right)+\frac{2}{M_{W}^{2}}(q \cdot k)\left(q \cdot \ell_{1}\right)\right]\right. \\
& \left.-m_{N}^{2}\left[\left(\ell_{2} \cdot \ell_{1}\right)+\frac{2}{M_{W}^{2}}\left(q \cdot \ell_{2}\right)\left(q \cdot \ell_{1}\right)\right]\right\}
\end{aligned}
$$

and

$$
\begin{aligned}
\Gamma\left(W^{+}\right. & \left.\rightarrow e^{+} e^{+} \mu^{-} \bar{\nu}_{\mu}\right)_{(L N V)} \\
= & \frac{64 \sqrt{2} G_{F}^{3}}{3} \frac{M_{W}^{5} m_{N}}{\Gamma_{N}} \\
& \times\left|U_{N e}\right|^{4} \int d \Phi_{2} \int d \Phi_{3} \frac{\left(k_{2} \cdot \ell_{2}\right)}{\left(M_{W}^{2}-m_{N}^{2}-2 k \cdot \ell_{2}\right)^{2}} \\
& \times\left\{\left(k_{1} \cdot \ell_{1}\right)+\frac{2}{M_{W}^{2}}\left(q \cdot k_{1}\right)\left(q \cdot \ell_{1}\right)\right\},
\end{aligned}
$$

where $d \Phi_{2}$ denotes the two-particle phase space of the first vertex and $d \Phi_{3}$ denotes the three-particle phase space of the $N$ decay [32].

Since a Dirac $N$ will only produce the LNC process while a Majorana $N$ will produce both LNC and LNV, we can find ways to detect the nature of $N$ by distinguishing between these two processes at the LHC. One cannot distinguish them by the rates because the mixings $\left|U_{N e}\right|$ and $\left|U_{N \mu}\right|$ are not known a priori, nor can we distinguish them by the final particles, because the final $\nu_{e}$ and $\bar{\nu}_{\mu}$ escape detection. However, due to the chiral character of the weak interactions and the fact that $\mu^{-}$is in a different 
fermion line (see Fig. 1), there will be a difference in the angular distribution of the muon momentum. Indeed, in the $N$ rest frame, if we define our $z$ axis along the initial $W^{+}$momentum $\mathbf{q}$, and we call $\theta$ the polar angle of the muon momentum $\mathbf{k}_{1}$ along this axis [i.e., $\left.\cos \theta=\mathbf{q} \cdot \mathbf{k}_{1} /\left(|\mathbf{q}|\left|\mathbf{k}_{1}\right|\right)\right]$, the angular distributions of the muon along the $W^{+}$direction in the $N$ rest frame are indeed different in the LNC and LNV processes. Calling $x_{N} \equiv m_{N} / M_{W}$, from Eqs. (2) and (3) we get

$$
\begin{aligned}
& \frac{d \Gamma}{d \cos \theta}\left(W^{+} \rightarrow e^{+} e^{+} \mu^{-} \nu_{e}\right)_{(L N C)} \\
& \quad=\mathcal{C} \times\left|U_{N e} U_{N \mu}\right|^{2}\left\{1-\cos \theta \times A\left(x_{N}\right)\left(\frac{2-x_{N}^{2}}{2+x_{N}^{2}}\right)\right\}
\end{aligned}
$$

and

$$
\begin{aligned}
& \frac{d \Gamma}{d \cos \theta}\left(W^{+} \rightarrow e^{+} e^{+} \mu^{-} \bar{\nu}_{\mu}\right)_{(L N V)} \\
& \quad=\mathcal{C} \times\left|U_{N e}\right|^{4}\left\{1-\cos \theta\left(\frac{2-x_{N}^{2}}{2+x_{N}^{2}}\right)\right\} .
\end{aligned}
$$

The global factor $\mathcal{C}$ is

$$
\mathcal{C} \equiv \frac{\sqrt{2}}{288(2 \pi)^{4}} G_{F}^{3} \frac{M_{W}^{8}}{\Gamma_{N}} \times F_{W}\left(x_{N}\right)
$$

where we defined the function

$$
\begin{aligned}
F_{W}\left(x_{N}\right) \equiv & \frac{\left(1-x_{N}^{2}\right)^{2}\left(2+x_{N}^{2}\right)}{x_{N}^{3}}\left\{\left(6-3 x_{N}^{2}-x_{N}^{4}\right) x_{N}^{2}\right. \\
& \left.+6\left(1-x_{N}^{2}\right) \ln \left(1-x_{N}^{2}\right)\right\},
\end{aligned}
$$

and where the neutrino width $\Gamma_{N}$ for $m_{N} \gtrsim 10 \mathrm{GeV}$ can be estimated as [24,27]

$$
\Gamma_{N} \simeq 1.1 \times \frac{G_{F}^{2}}{12 \pi^{3}} M_{W}^{5} F_{N}\left(x_{N}\right) \sum_{\ell}\left|U_{N \ell}\right|^{2}
$$

where we have included the function $F_{N}\left(x_{N}\right)$,

$$
\begin{aligned}
F_{N}\left(x_{N}\right)= & \frac{2}{x_{N}^{3}}\left\{\left(6-3 x_{N}^{2}-x_{N}^{4}\right) x_{N}^{2}\right. \\
& \left.+6\left(1-x_{N}^{2}\right) \ln \left(1-x_{N}^{2}\right)\right\}
\end{aligned}
$$

This function $F_{N}(x)$ is due to the momentum dependence of the $W$ propagator, and is an improvement in the $m_{N}$ dependence of $\Gamma_{N}$ estimate of Refs. [24,27], where the $W$ propagator was taken as a point interaction. In the limit $x_{N} \ll 1, F_{W}\left(x_{N}\right) / F_{N}\left(x_{N}\right) \rightarrow 1$.

In turn, the factor $A\left(x_{N}\right)$ of the angle-dependent term in the LNC distribution is given by

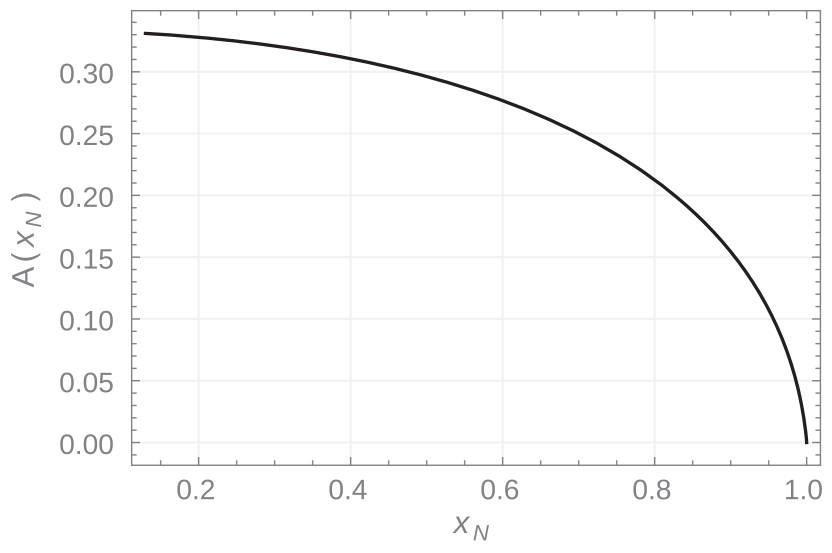

FIG. 2. Analyzing power function $A\left(x_{N}\right)$ given in Eq. (10). $x_{N}=m_{N} / M_{W}$.

$A\left(x_{N}\right)=\left(x_{N}^{2}-1\right) \frac{\left(6-x_{N}^{2}\right) x_{N}^{2}+\left(6-4 x_{N}^{2}\right) \ln \left(1-x_{N}^{2}\right)}{\left(6-3 x_{N}^{2}-x_{N}^{4}\right) x_{N}^{2}+6\left(1-x_{N}^{2}\right) \ln \left(1-x_{N}^{2}\right)}$

and it is called the analyzing power, as it modulates the angular dependence of the distribution [14,31]. In the limit $x_{N} \ll 1, A\left(x_{N}\right) \rightarrow 1 / 3$, while for $x_{N} \rightarrow 1, A\left(x_{N}\right) \rightarrow 0$. In Fig. 2 we show the behavior of $A\left(x_{N}\right)$ as a function of $x=m_{N} / M_{W}$.

The difference between the LNC and LNV angular distributions of Eqs. (4) and (5) is essentially the analyzing power factor $A\left(x_{N}\right)$ in the LNC expression. This difference can be explained as follows. The neutrino $N$ produced in the decay $W^{+} \rightarrow e^{+} N$ must be of left-handed chirality because of the weak current (in the same way, the produced positron in this decay must be right-handed).

Provided that $m_{N}$ is considerably smaller than $M_{W}$, the helicity of $N$ will be mostly left-handed as well (in the $N$ rest frame, the $N$ spin will be polarized along the $W$ momentum q; see Fig. 3). For the calculation of the primary process $W^{+} \rightarrow e^{+} N$, where $N$ has a polarization vector $s^{\mu}$ (this vector satisfies $s \cdot s=-1$ and $s \cdot k=0$, where $k$ is the $N$ momentum), one obtains the following squared matrix element:

$$
\begin{aligned}
|\overline{\mathcal{M}}|^{2}= & \frac{4 M_{W}^{2} G_{F}}{3 \sqrt{2}}\left|U_{N \ell}\right|^{2}\left((k \cdot \ell)+\frac{2}{M_{W}^{2}}(q \cdot k)(q \cdot \ell)\right. \\
& \left.-m_{N}(s \cdot \ell)-\frac{2 m_{N}}{M_{W}^{2}}(q \cdot s)(q \cdot \ell)\right) .
\end{aligned}
$$

Given a neutrino momentum $k=(E, 0,0, \mathrm{k})$, the right and left helicities of $N$ correspond to $s= \pm(\mathrm{k}, 0,0, E) / m_{N}$,

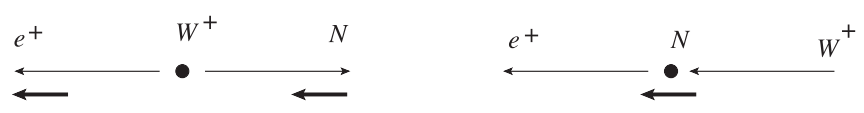

FIG. 3. $W^{+} \rightarrow e^{+} N$ in two frames: the $W$ rest frame (left) and the $N$ rest frame (right). Notice that the $N$ spin direction is conserved under the boost. 
respectively. It is then easy to show that the probabilities of the two helicities are in the ratio $|\overline{\mathcal{M}}|_{\text {right }}^{2}:|\overline{\mathcal{M}}|_{\text {left }}^{2}=$ $m_{N}^{2}: 2 M_{W}^{2}$, and so the probabilities to produce $N$ with right-handed or left-handed helicity are

$$
P_{\text {right }}=\frac{m_{N}^{2}}{2 M_{W}^{2}+m_{N}^{2}}, \quad P_{\text {left }}=\frac{2 M_{W}^{2}}{2 M_{W}^{2}+m_{N}^{2}} .
$$

Now, in the decay of a polarized $N$, the polarization vector sets a direction along which an anisotropic muon emission occurs (for an unpolarized $N$ the muon emission is isotropic). Moreover, since the muon comes from a different weak current in the LNC and LNV processes (see Fig. 1), the anisotropic distributions in these two processes are different. We must point out that the predominant lefthanded polarization of the produced $N$ occurs regardless of its Dirac or Majorana nature. The latter has an effect in the subsequent decay of $N$. For a Majorana $N$, both decays

$$
\begin{gathered}
N \rightarrow \mu^{-} e^{+} \nu_{e}, \\
N \rightarrow e^{+} \mu^{-} \bar{\nu}_{\mu}
\end{gathered}
$$

are allowed, while for a Dirac $N$ produced in $W^{+} \rightarrow e^{+} N$, the latter mode is forbidden.

Now, for a polarized $N$ these decays have the following $\theta$ distributions:

$\frac{d \Gamma}{d \cos \theta}\left(N_{\mathrm{pol}} \rightarrow \mu^{-} e^{+} \nu_{e}\right) \sim\left|U_{N \mu}\right|^{2}\left\{1+A\left(x_{N}\right) \cos \theta\right\}$

and

$$
\frac{d \Gamma}{d \cos \theta}\left(N_{\mathrm{pol}} \rightarrow e^{+} \mu^{-} \bar{\nu}_{\mu}\right) \sim\left|U_{N e}\right|^{2}\{1-\cos \theta\},
$$

where $\theta$ is, as before, the angle between the momentum of the muon and the polarization vector of $N$ at rest. Here it is clear that the two angular distributions are different precisely because the muon is attached to a different fermion line in the weak interaction. Now, to get to the distributions of the full LNC and LNV processes of Eqs. (4) and (5) we must weigh the polarized $N$ decays by the probabilities in Eq. (12) of the two longitudinal polarizations produced in the primary decay $W \rightarrow e^{+} N$. The result coincides with the distributions of Eqs. (4) and (5).

Given the fact that the LNC and LNV muon angular distributions are different, we propose to use the following forward-backward asymmetry to try to distinguish between the Dirac vs Majorana character of the intermediate neutrino $N$ that induces the events:

$$
\begin{aligned}
A_{F B} & =\frac{N(\cos \theta>0)-N(\cos \theta<0)}{N(\cos \theta>0)+N(\cos \theta<0)} \\
& =\frac{\int_{0}^{1} \frac{d \Gamma}{d \cos \theta} d(\cos \theta)-\int_{-1}^{0} \frac{d \Gamma}{d \cos \theta} d(\cos \theta)}{\int_{-1}^{1} \frac{d \Gamma}{d \cos \theta} d(\cos \theta)},
\end{aligned}
$$

where $N(\cos \theta>0)$ and $N(\cos \theta<0)$ denote the number events with the muon moving forwards or backwards with respect to the decaying $W$, in the $N$ rest frame of the heavy neutrino.

If $N$ is a Dirac neutrino, only the LNC process occurs, in which case the analyzing power $A\left(m_{N} / M_{W}\right)$ directly determines the outcome of the experimental forward-backward asymmetry, regardless of lepton mixing (although the mixing determines the total number of events):

$$
A_{F B}^{(\text {Dirac) }}=-\frac{1}{2} A\left(x_{N}\right)\left(\frac{2-x_{N}^{2}}{2+x_{N}^{2}}\right) .
$$

Instead, if $N$ is a Majorana neutrino, the rate of events $W \rightarrow e^{+} e^{+} \mu^{-} \nu$ is the sum of the LNC and LNV rates given in Eqs. (4) and (5), and then the forward-backward asymmetry will depend not only on the analyzing power of the LNC component, but also on the relative lepton mixing elements $\left|U_{N e}\right|$ and $\left|U_{N \mu}\right|$ :

$$
A_{F B}^{(\mathrm{Maj})}=-\frac{A\left(x_{N}\right)\left|U_{N \mu}\right|^{2}+\left|U_{N e}\right|^{2}}{2\left(\left|U_{N \mu}\right|^{2}+\left|U_{N e}\right|^{2}\right)}\left(\frac{2-x_{N}^{2}}{2+x_{N}^{2}}\right) .
$$

The Dirac asymmetry (18) is independent of the mixings and, due to the function $A\left(x_{N}\right)$ (see Fig. 2), is approximatively $-1 / 6$ for a wide range of $m_{N}$, except if $m_{N}$ approaches $M_{W}$, in which case it tends to vanish.

In contrast, the Majorana asymmetry (19) does depend on the mixings and in general it is larger than in the Dirac case. For events $e^{+} e^{+} \mu^{-}$, if $\left|U_{N \mu}\right| \gg\left|U_{N e}\right| \mid$, it tends to the same value as in the Dirac case because it is dominated by the LNC contribution, but in the opposite limit (namely, $\left.\left|U_{N \mu}\right| \ll\left|U_{N e}\right|\right)$ dominated by the LNV contribution, it is larger - close to $-1 / 2$ for most values of $m_{N}$, and decreasing to $-1 / 6$ as $m_{N}$ approaches $M_{W}$.

Consequently, one can in principle distinguish the Dirac from the Majorana case using this asymmetry. However, notice that in the case $\left|U_{N \mu}\right| \gg\left|U_{N e}\right|$, for events $e^{ \pm} e^{ \pm} \mu^{\mp}$ the asymmetry is the same for both Dirac and Majorana $N$, because the Majorana asymmetry is dominated by the LNC contribution.

In this case the channels $\mu^{ \pm} \mu^{ \pm} e^{\mp}$ are more appropriate for discrimination, because now the Majorana asymmetry is dominated by the LNV contribution. The angular distribution in this case is that of the electron flavor instead of the muon.

\section{DISTINGUISHING THE SAME-SIGN LEPTONS AT THE LHC}

In order to experimentally determine the asymmetries of Eqs. (18) and (19), one needs to identify which of the two equal-sign leptons originates from the first vertex and from the second vertex (see Fig. 1). From this identification one can apply the appropriate boosts to the $W$ rest frame or to 

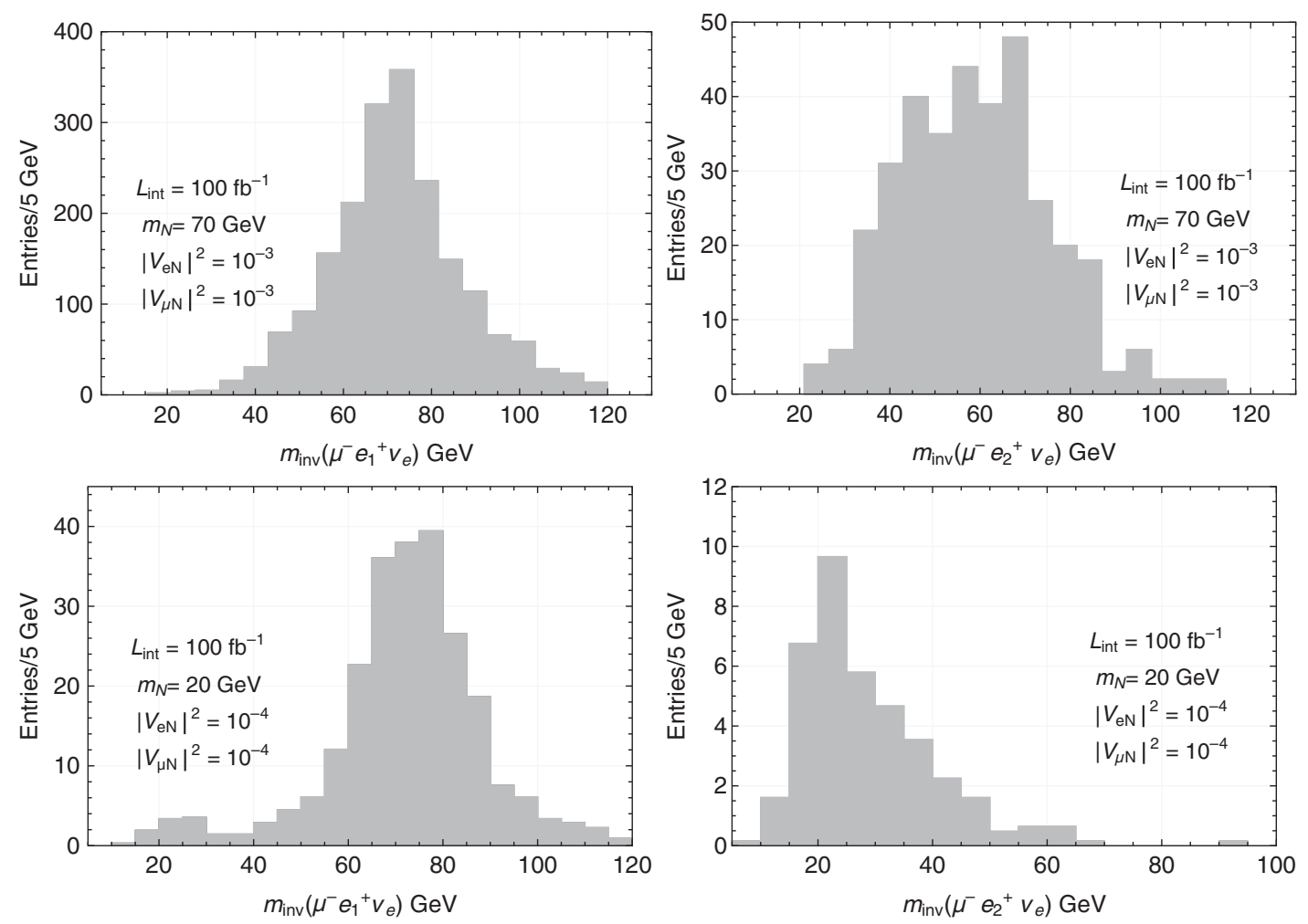

FIG. 4. Invariant mass distributions for the $e_{1}^{+} \mu^{-} \nu_{e}$ and $e_{2}^{+} \mu^{-} \nu_{e}$ invariant masses and for several heavy neutrino masses with a centerof-mass energy of $\sqrt{s}=13 \mathrm{TeV}$.

the $N$ rest frame, as required. In this section we study this issue of discriminating the two equal-sign leptons (see also Ref. [28]).

As an example, consider the LNC production and decay channel $p p \rightarrow W^{+} \rightarrow e^{+} N \rightarrow e^{+} \mu^{-} e^{+} \nu_{e}$, in which two positrons are in the final state. In the LNV channel there is one $\bar{\nu}_{\mu}$ in the final state, but (as we shall see) the considerations we use apply to the latter case as well.

In Fig. 4 we show the invariant masses for the $e_{1}^{+} \mu^{-} \nu_{e}$ and $e_{2}^{+} \mu^{-} \nu_{e}$, where $e_{1}^{+}$and $e_{2}^{+}$are the harder and softer positron sorted by energy, respectively. The events were generated at leading order using MADGRAPH 5 [33], hadronized with Pythia 6 [34], and passed to DelPhES 3 [35] for detector simulation. In the figure we can see that when the heavy neutrino mass is above $70 \mathrm{GeV}$, it is better reconstructed by the hardest positron. This is in agreement with the expectation that in this case the heavy neutrino carries most of the energy.

Conversely, when the heavy neutrino mass is below $40 \mathrm{GeV}$, it is the softer positron that reconstructs the heavy neutrino mass, as can be seen in Fig. 4. Once again, this is in agreement with the expectation that the heavy neutrino carries less energy than in the more massive case, since in the massless limit is would have exactly half of the energy taken from the $W$ decay. We find that in the region between 50-70 GeV both electrons show similar behavior in the $m_{N}$ reconstruction and hence cannot be distinguished with the above strategy. Another interesting region is where $m_{N} \leq 10 \mathrm{GeV}$. In this region the decay length of the heavy neutrino becomes visible in the form of a displaced vertex inside the detector. Clearly, in this case the distinction of both positrons will be obvious. The background processes and the possibility of distinguishing both same-sign leptons have been extensively studied in Refs. [28-30]. Therefore we do not discuss this issue further here. In summary, for $m_{N} \lesssim 10 \mathrm{GeV}$, the two leptons can be separated due to vertex displacement. For higher masses no vertex displacement is observable since $N$ is too short lived. In those cases the separation of the two leptons is less certain. As shown, statistically the primary lepton tends to be the most energetic one if $m_{N} \lesssim 50 \mathrm{GeV}$ and the less energetic one if $m_{N} \gtrsim 70 \mathrm{GeV}$. In the mass region between 50 and $70 \mathrm{GeV}$ no such distinction occurs.

Another important experimental issue here is the expected number of events. For a realistic analysis that involves angular distributions, one would need a rather large sample of these rare events. According to Ref. [36], at the end of the LHC Run II, with an integrated luminosity of $120 \mathrm{fb}^{-1}$, ATLAS is expected to have of the order of $2 \times$ $10^{8}$ leptonic $W$ decays into $e \nu$ and $\mu \nu$. Considering that the $W$ width is $\Gamma_{W} \sim 2 \mathrm{GeV}$ and the branching ratio into each lepton is near 10\%, using Eqs. (4)-(6) and (8) we can estimate the expected number of $W \rightarrow e^{+} e^{+} \mu^{-} \nu$ or $W \rightarrow$ $\mu^{+} \mu^{+} e^{-} \nu$ events. Fake leptons which mainly originate from 
heavy-flavor meson decays represent the leading background. The main contributions to this background process come from $\gamma^{*} / Z+$ jets and $t \bar{t}$ and have been fully characterized in Refs. [26,28,37]. The WZ background contribution is found to be subdominant. For instance, using the cuts proposed in Table II of Ref. [28], and summing the backgrounds indicated there, at the LHC one expects three background events for $120 \mathrm{fb}^{-1}$ of integrated luminosity. Then, without considering the impact of systematic errors, the expected number of events for the Majorana and Dirac scenarios are estimated to be

$$
\begin{aligned}
& N\left(W \rightarrow e^{+} e^{+} \mu^{-} \nu\right)_{\text {Maj }} \\
& \sim 6 \times 10^{6}\left(\left|U_{N e}\right|^{2}+\left|U_{N \mu}\right|^{2}\right)\left|U_{N e}\right|^{2} / \sum_{l}\left|U_{N l}\right|^{2}, \\
& N\left(W \rightarrow e^{+} e^{+} \mu^{-} \nu\right)_{\text {Dirac }} \\
& \sim 6 \times 10^{6}\left|U_{N e} U_{N \mu}\right|^{2} / \sum_{l}\left|U_{N l}\right|^{2} .
\end{aligned}
$$

From these expressions one can deduce that the largest of the mixings $\left|U_{N e}\right|^{2}$ and $\left|U_{N \mu}\right|^{2}$ must be at least of order $10^{-6}$ for the LHC Run II to get a positive signal of these modes. Take, for example, the case $\left|U_{N e}\right| \gg\left|U_{N \mu}\right| \sim\left|U_{N \tau}\right|$; then, the above reduces to

$$
\begin{gathered}
N\left(W \rightarrow e^{+} e^{+} \mu^{-} \nu\right)_{\text {Maj }} \sim 6 \times 10^{6}\left|U_{N e}\right|^{2}, \\
N\left(W \rightarrow e^{+} e^{+} \mu^{-} \nu\right)_{\text {Dirac }} \sim 6 \times 10^{6}\left|U_{N \mu}\right|^{2},
\end{gathered}
$$

implying that the expected number of these events for $\left|U_{N e}\right|^{2} \sim 10^{-6}$ is six (zero) for Majorana (Dirac) $N$.

Alternatively, if $\left|U_{N e}\right| \sim\left|U_{N \mu}\right| \sim\left|U_{N \tau}\right|$, the above reduces to

$$
\begin{gathered}
N\left(W \rightarrow e^{+} e^{+} \mu^{-} \nu\right)_{\text {Maj }} \sim 4 \times 10^{6}\left|U_{N e}\right|^{2}, \\
N\left(W \rightarrow e^{+} e^{+} \mu^{-} \nu\right)_{\text {Dirac }} \sim 2 \times 10^{6}\left|U_{N e}\right|^{2},
\end{gathered}
$$

and the expected number of events for $\left|U_{N e}\right|^{2} \sim 10^{-6}$ would be around four (two) for Majorana (Dirac) $N$.

Finally, if $\left|U_{N \mu}\right| \gg\left|U_{N e}\right| \sim\left|U_{N \tau}\right|$, the number of $e^{ \pm} e^{ \pm} \mu^{\mp}$ events for both Dirac and Majorana cases are much smaller, in which case the $\mu^{ \pm} \mu^{ \pm} e^{\mp}$ events should be considered. Therefore, for an integrated luminosity of $120 \mathrm{fb}^{-1}$ at the LHC Run II, $W \rightarrow e^{ \pm} e^{ \pm} \mu^{\mp}$ events can be found provided $\left|U_{N e}\right|^{2} \gtrsim 10^{-6}$, while $W \rightarrow \mu^{ \pm} \mu^{ \pm} e^{\mp}$ signals can be found if $\left|U_{N \mu}\right|^{2} \gtrsim 10^{-6}$. These are the limits in cases where $m_{N}<M_{W}$, but as $m_{N}$ approaches $M_{W}$ these theoretical rates are smaller and the lowest $\left|U_{N \ell}\right|^{2}$ for detection becomes larger. Moreover, in cases where
$\left|U_{N e}\right|^{2} \sim\left|U_{N \mu}\right|^{2}$, the discrimination between Dirac and Majorana $N$ requires the forward-backward asymmetry of Eq. (17). In order to build this asymmetry one needs more data than the minima deduced above; hence, $\left|U_{N \ell}\right|^{2}$ must be above $10^{-6}$ or the $W \rightarrow \ell \nu$ data sample must be larger than what is expected at the end of the LHC Run II. On the other hand, current upper bounds for $\left|U_{N e}\right|^{2}$ and $\left|U_{N \mu}\right|^{2}$ for $N$ with a mass in the range $10 \mathrm{GeV}<m_{N}<$ $M_{W}$ are near $10^{-5}$ [38], leaving about an order of magnitude in the range of the mixings to explore in the LHC Run II.

\section{CONCLUSIONS}

We presented a new method to discriminate Dirac vs Majorana sterile neutrinos with masses $m_{N} \leq m_{W}$ that would induce $W$ trilepton decays $e^{ \pm} e^{ \pm} \mu^{\mp}$ and $\mu^{ \pm} \mu^{ \pm} e^{\mp}$ at the LHC. A simple strategy based on a forwardbackward asymmetry was proposed. This asymmetry is different depending on the Dirac/Majorana character of the heavy neutrino. If the heavy neutrino is a Dirac particle, the forward-backward asymmetry depends on the heavy neutrino mass but not on the heavy-to-light mixing parameters. Instead, for a Majorana neutrino the asymmetry depends on both the mass and the mixing matrix elements. The method presented here is complementary to the strategy proposed in Refs. [28-30], in the sense that it allows to distinguish the neutrino nature also in the case where the neutrinolepton mixing elements are of the same order.

In order to construct the asymmetry, a crucial point is to identify the primary and secondary same-charge leptons in the final state $\ell^{ \pm} \ell^{ \pm} \ell^{\mp \mp}$. Using invariant mass analysis distributions, we found ranges for $m_{N}$ where the LHC could distinguish among these two leptons.

If $m_{N} \lesssim 50 \mathrm{GeV}\left(m_{N} \gtrsim 70 \mathrm{GeV}\right)$, the first positron tends to be the most (least) energetic, while in the region $50 \lesssim m_{N} \lesssim 70 \mathrm{GeV}$, the order of the two same-sign leptons cannot be distinguished.

We estimated the number of these events for the integrated luminosity of $120 \mathrm{fb}^{-1}$ expected at the end of the LHC Run II, and found that both Majorana and Dirac signals could be found provided that at least one of the mixings $\left|U_{N \mu}\right|^{2}$ or $\left|U_{N e}\right|^{2}$ is $10^{-6}$ or above.

\section{ACKNOWLEDGMENTS}

J. V. thanks Goran Senjanović for useful and enlightening discussions. This work is supported in part by Fondecyt (Chile) Grants No. 3170154, No. 1180232 and No. 1170171, and by CONICYT (Chile) Ring ACT1406 and PIA/Basal FB0821. 
[1] Y. Fukuda et al. (Super-Kamiokande Collaboration), Evidence for Oscillation of Atmospheric Neutrinos, Phys. Rev. Lett. 81, 1562 (1998).

[2] Q. R. Ahmad et al. (CNO Collaboration), Direct evidence for Neutrino Flavor Transformation from Neutral Current Interactions in the Sudbury Neutrino Observatory, Phys. Rev. Lett. 89, 011301 (2002).

[3] K. Eguchi et al. (KamLAND Collaboration), First Results from KamLAND: Evidence for Reactor Anti-Neutrino Disappearance, Phys. Rev. Lett. 90, 021802 (2003).

[4] D. V. Forero, M. Tortola, and J. W. F. Valle, Neutrino oscillations refitted, Phys. Rev. D 90, 093006 (2014).

[5] E. Majorana, Teoria simmetrica dell'elettrone e del positrone, Il Nuovo Cimento 14, 171 (1937).

[6] P. Minkowski, $\mu \rightarrow e \gamma$ at a rate of one out of 109 muon decays?, Phys. Lett. B 67, 421 (1977).

[7] R. N. Mohapatra and G. Senjanović, Neutrino Mass and Spontaneous Parity Violation, Phys. Rev. Lett. 44 (1980) 912.

[8] S. L. Glashow, The future of elementary particle physics, NATO Sci. Ser. B 61, 687 (1980).

[9] M. Gell-Mann, P. Ramond, and R. Slansky, Complex spinors and unified theories, Conference Proceedings C790927, 315 (1979).

[10] T. Yanagida, Horizontal symmetry and masses of neutrinos, Conference Proceedings C7902131, 95 (1979).

[11] W.-Y. Keung and G. Senjanović, Majorana Neutrinos and the Production of the Right-Handed Charged Gauge Boson, Phys. Rev. Lett. 50, 1427 (1983).

[12] A. Ferrari, J. Collot, M.-L. Andrieux, B. Belhorma, P. de Saintignon, J.-Y. Hostachy, Ph. Martin, and M. Wielers, Sensitivity study for new gauge bosons and right-handed Majorana neutrinos in $p p$ collisions at $s=14 \mathrm{TeV}$, Phys. Rev. D 62, 013001 (2000).

[13] S. Kovalenko, Z. Lu, and I. Schmidt, Lepton number violating processes mediated by Majorana neutrinos at hadron colliders, Phys. Rev. D 80, 073014 (2009).

[14] T. Han, I. Lewis, R. Ruiz, and Z.-g. Si, Lepton number violation and $W^{\prime}$ chiral couplings at the LHC, Phys. Rev. D 87, 035011 (2013).

[15] A. Das and N. Okada, Improved bounds on the heavy neutrino productions at the LHC, Phys. Rev. D 93, 033003 (2016).

[16] F. F. Deppisch, P. S. Bhupal Dev, and A. Pilaftsis, Neutrinos and collider physics, New J. Phys. 17, 075019 (2015).

[17] A. Das, P. Konar, and S. Majhi, Production of heavy neutrino in next-to-leading order QCD at the LHC and beyond, J. High Energy Phys. 06 (2016) 019.

[18] A. Das, P. Konar, and A. Thalapillil, Jet substructure shedding light on heavy Majorana neutrinos at the LHC, J. High Energy Phys. 02 (2018) 083.

[19] A. Das, Pair production of heavy neutrinos in next-toleading order QCD at the hadron colliders in the inverse seesaw framework, arXiv:1701.04946.

[20] A. Das, P. S. B. Dev, and C. S. Kim, Constraining sterile neutrinos from precision Higgs data, Phys. Rev. D 95, 115013 (2017).
[21] A. Das, P. S. B. Dev, and R. N. Mohapatra, Same sign vs opposite sign dileptons as a probe of low scale seesaw mechanisms, Phys. Rev. D 97, 015018 (2018).

[22] G. Aad et al. (ATLAS Collaboration), Search for heavy Majorana neutrinos with the ATLAS detector in $p p$ collisions at $\sqrt{s}=8 \mathrm{TeV}$, J. High Energy Phys. 07 (2015) 162.

[23] V. Khachatryan et al. (CMS Collaboration), Search for heavy Majorana neutrinos in $\mu^{ \pm} \mu^{ \pm}+$jets events in protonproton collisions at $\sqrt{s}=8 \mathrm{TeV}$, Phys. Lett. B 748, 144 (2015).

[24] A. Atre, T. Han, S. Pascoli, and B. Zhang, The search for heavy Majorana neutrinos, J. High Energy Phys. 05 (2009) 030.

[25] J. C. Helo, M. Hirsch, and S. Kovalenko, Heavy neutrino searches at the LHC with displaced vertices, Phys. Rev. D 89, 073005 (2014).

[26] E. Izaguirre and B. Shuve, Multilepton and lepton jet probes of sub-weak-scale right-handed neutrinos, Phys. Rev. D 91, 093010 (2015).

[27] C. O. Dib and C. S. Kim, Discovering sterile neutrinos ligther than $M_{W}$ at the LHC, Phys. Rev. D 92, 093009 (2015).

[28] C. O. Dib, C. S. Kim, K. Wang, and J. Zhang, Distinguishing Dirac/Majorana sterile neutrinos at the LHC, Phys. Rev. D 94, 013005 (2016).

[29] C. O. Dib, C. S. Kim, and K. Wang, Signatures of Dirac and Majorana sterile neutrinos in trilepton events at the LHC, Phys. Rev. D 95, 115020 (2017).

[30] C. O. Dib, C. S. Kim, and K. Wang, Search for heavy sterile neutrinos in trileptons at the LHC, Chin. Phys. C 41, 103103 (2017).

[31] T. M. P. Tait and C. P. Yuan, Single top quark production as a window to physics beyond the standard model, Phys. Rev. D 63, 014018 (2000).

[32] C. Patrignani et al. (Particle Data Group), Review of particle physics, Chin. Phys. C 40, 100001 (2016).

[33] J. Alwall, R. Frederix, S. Frixione, V. Hirschi, F. Maltoni, O. Mattelaer, H.-S. Shao, T. Stelzer, P. Torrielli, and M. Zaro, The automated computation of tree-level and next-to-leading order differential cross sections, and their matching to parton shower simulations, J. High Energy Phys. 07 (2014) 079.

[34] T. Sjostrand, S. Mrenna, and P.Z. Skands, PythiA 6.4 physics and manual, J. High Energy Phys. 05 (2006) 026.

[35] J. de Favereau, C. Delaere, P. Demin, A. Giammanco, V. Lemaître, A. Mertens, and M. Selvaggi (DELPHES 3 Collaboration), Delphes 3, A modular framework for fast simulation of a generic collider experiment, J. High Energy Phys. 02 (2014) 057.

[36] G. Aad et al. (ATLAS Collaboration), Measurement of $W^{ \pm}$ and Z-boson production cross sections in $p p$ collisions at $\sqrt{s}=13 \mathrm{TeV}$ with the ATLAS detector, Phys. Lett. B 759, 601 (2016).

[37] A. M. Sirunyan et al. (CMS Collaboration), Search for heavy neutral leptons in events with three charged leptons in protonproton collisions at $\sqrt{s}=13 \mathrm{TeV}$, arXiv:1802.02965.

[38] P. Abreu et al. (DELPHI Collaboration), Search for neutral heavy leptons produced in $Z$ decays, Z. Phys. C 74, 57 (1997). 